\title{
«Por un trabajo digno» - Emergencia y representaciones de un movimiento de niñas, niños y adolescentes trabajadores en Bolivia
}

\author{
Alexandre Rutigliano*
}

\begin{abstract}
- Licenciado en Antropología Cultural, y magíster en Derechos Humanos y Acción Humanitaria por la Escuela de París de Relaciones Internacionales (Paris School of International Affairs, PSIA) de SciencesPo Paris. Actualmente, es consultor en políticas públicas educativas para la Organización para la Cooperación y el Desarrollo Económico (OCDE) localizada en París. Correo electrónico: alexandre.rutigliano@gmail.com. https://orcid.org/0000-0002-8706-4781
\end{abstract}

Fecha de recepción: 04/11/2019. Fecha de aceptación: 23/06/2020. 


\title{
«Por un trabajo digno» - Emergencia y representaciones de un movimiento de niñas, niños y adolescentes trabajadores en Bolivia
}

\section{RESUMEN}

Desde que las primeras organizaciones de niños, niñas y adolescentes trabajadores (NNAT) emergieron en los años 1980 en Bolivia, emprendieron acciones con y contra el Estado, e incluso colaboraron en la redacción de la Nueva Constitución (2008-2009) y del Nuevo Código Niña Niño Adolescente (2013-2014). Como movimiento social, han luchado por sus derechos a trabajar, a tener protecciones legales específicas por ser niños y niñas trabajadores y a ser incluidos en el diseño de leyes y políticas que los impactan. Como parte de un trabajo etnográfico más amplio, este artículo se enfoca en la historia de las representaciones de la infancia y del trabajo que son todavía dominantes en la mayoría de las organizaciones internacionales, y en cómo algunos varios NNAT bolivianos se enfrentan a ellas y definen derechos más contextualizados e inclusivos. De manera general, este trabajo muestra que no hay un ser niño universal, y que los niños, niñas y adolescentes se deben considerar como actores en los espacios social y político.

Palabras clave: Bolivia, movimientos sociales, derechos humanos, trabajo infantil, derecho a la participación.

\section{«For a dignified work» - Emergency and representations of a movement of working children and adolescents in Bolivia}

\begin{abstract}
Since the first organisations of working children and adolescents (NNAT) emerged in the 1980's in Bolivia, they have been leading ongoing actions in collaboration with and against the State, including the writing of the New Constitution in 2008-9 and of the New Child and Adolescents Code in 2013-14. As a social movement, they have been fighting for their rights to work, to have special legal protections for being working children and to be involved in the design of laws and policies that affect them. As part of a broader ethnographic investigation, this article focuses on the history of the the representations of childhood and work that are still today dominant in most international organisations, and on how some bolivian NNAT confront them and define more contextualised and inclusive rights. Overall, this work shows that there is no universal way of being a child, and that children and adolescents must be considered as actors in the social and political spheres.
\end{abstract}

Keywords: Bolivia, social movements, human rights, child labour, right to participation. 


\section{INTRODUCCIÓN}

Les daría alas a los niños, pero dejaría que ellos aprendieran a volar por si mismos Gabriel García Márquez

La Unión Boliviana de Niñas, Niños y Adolescentes Trabajadores (Unatsbo) emergió al inicio del siglo y estuvo involucrada en los mayores eventos políticos de la historia reciente de Bolivia. Desde la aparición de las primeras organizaciones de niñas, niños y adolescentes trabajadores (NNAT) en el país en los años 1990, estos han luchado continuamente contra el Estado - y, en algunas ocasiones, en colaboración con él一, para defender sus derechos y mejorar su situación.

En dos décadas lograron una organización propia y, a través de varias acciones colectivas y de la constitución de un objetivo común, consiguieron un espacio de participación en la redacción de la Nueva Constitución Política del Estado Plurinacional de Bolivia en 2008. Luego, en 2013, las organizaciones de NNAT de todos los departamentos del país llegaron a La Paz para manifestarse en la Plaza Murillo, el mayor símbolo político del Estado andino. Esta manifestación fue una reacción detonada por el anuncio del gobierno sobre el nuevo Código Niña Niño Adolescente (CNNA), en el cual las demandas de los niños, niñas y adolescentes no fueron inicialmente tenidas en cuenta. Después de violentos altercados con la policía, provocando incluso un choque ampliamente mediatizado, representantes de NNAT consiguieron una reunión con el presidente Evo Morales —él mismo ex NNAT—. De esta manera, consiguieron restablecer las negociaciones con el Estado, para lograr finalmente integrar partes de sus demandas en el nuevo CNNA, incluso la reducción de la edad mínima para trabajar que constituye un punto central de su lucha. Hoy, el CNNA establece uno de los marcos legales más extendidos de la región de Latinoamérica en términos de protección de niños, niñas y adolescentes trabajadores.

A través de una investigación etnográfica de cinco meses, se exploró el nacimiento y la consolidación de una organización local de NNAT en Bolivia, la organización Taypinats en La Paz. Se pasaron más de cinco meses con un grupo de niñas, nińos y adolescentes actores en los espacios sociales y políticos de la capital política de Bolivia, con el objetivo de aclarar cómo consiguieron organizarse en un movimiento social nacional estructurado y continuo.

Este artículo se inscribe en la continuidad de una tesis de maestría realizada entre mayo de 2018 y febrero de 2019, con un terreno etnográfico que se extendió de junio a octubre de 2018. Enfocándose en una organización local, trata de entender el proceso histórico y político de construcción del movimiento social de NNAT boliviano en el país, la Unión de Niños y Niñas Adolescentes Trabajadores de Bolivia (Unatsbo). 
Este trabajo desea dar justicia a esos NNAT que han luchado desde hace más de treinta años por sus derechos. Después de breves aclaraciones sobre la metodología, toma primero un enfoque sociohistórico, alimentado por un trabajo de archivos, para entender cómo y en cuál contexto nació Taypinats. A través de la presentación de los niños y jóvenes y del trabajo etnográfico, intenta después explicar cómo llegaron a una conciencia y movilización colectivas, es decir, a ser NNAT como identidad de grupo y, a menudo, politizada. Finalmente, la última parte de este artículo se enfoca en el tema de la relatividad de las representaciones sobre la infancia, analizando las nociones de «niño trabajador» y «protagonista» y esbozando una crítica de la visión occidental del ser niño.

Por lo tanto, a través del análisis de la gestación de Taypinats, su transformación en organización y su implicación en un movimiento social, esta investigación apunta a mostrar la representación del trabajo y del ser niño de estos nińos, niñas y adolescentes, un ser niño específico al contexto boliviano, aunque hace eco a la situación de grupos de NNAT en otros países latinoamericanos. El objetivo subyacente es, entonces, esbozar un cuestionamiento sobre los límites y contradicciones del paradigma occidental sobre el trabajo infantil que han construido algunas organizaciones internacionales.

Sin pretensión de encontrar una solución a los problemas implicados en el tema del trabajo infantil, se quiere dar una voz a los NNAT en los debates. Es decir que, reconociendo que encontrar una solución potencial al trabajo infantil no es simple y unilateral, se desea contribuir a una literatura reciente que analiza los derechos de los niños, y los derechos humanos en general, como dependiendo del contexto en el cual emergen (Truchon \& Saillant, 2013), y que su realización no se puede lograr sin la participación de los individuos interesados.

\section{CONSIDERACIONES METODOLÓGICAS}

La literatura relativa a los movimientos sociales es abundante y diversa, y ha generado debates considerables en las ciencias sociales. Gracias en gran parte a la contribución del sociólogo estadounidense Charles Tilly desde los años 1970, al análisis se centralizó en las dimensiones políticas e históricas de los movimientos sociales (Alonso, 2012). Para evitar la fragmentación teórica que caracteriza esta literatura y lograr una comprensión más compleja de la solución abordada por las organizaciones de NNAT, este trabajo adopta un marco bastante amplio. Se trata de entender la movilización colectiva como un resultado del proceso de la articulación de factores económicos, sociales, políticos e individuales que llevan a un grupo de individuos a contestar expresiones de la autoridad bajo una identificación común. Asimismo, este análisis se sitúa en un marco pluridisciplinar y un movimiento de 
autores que consideran que se necesita «una metodología que dé la prioridad al análisis del ambiente político de las movilizaciones, de las interacciones entre grupos contestatarios y las autoridades, de sus modos de acciones, todo ello basándose sobre observaciones de terrenos que no se limitan a las direcciones de las organizaciones de movimiento social» (Goirand, 2010, p. 11). La perspectiva etnográfica permitió entender el proceso complejo que llevó a la construcción de conciencia de grupo y de identificación de los NNAT, así como la interacción de esos actores sociales con el Estado, que más allá de estar solo marcada por la confrontación, se caracteriza por una importante cooperación inscrita en la historia política de Bolivia.

Con el tiempo, los NNAT llegaron a ser un movimiento social que reúne todas las características descritas por autores tal como Tilly, es decir valor, unidad, número [de partidarios] y compromiso (Tilly, en Goirand, 2010, p. 16) . Además, como escribieron Tilly y Wood (2003) hablando de un movimiento social en Francia, «a pesar de innovaciones y variaciones continuas de pequeńa escala de una estructura política a la otra, el movimiento social se desarrolló y se difundió como un todo conectado. En este sentido, el movimiento social tiene una historia» (p. 3). Así pues, es importante entender la historia de Taypinats y Unatsbo como organizaciones interconectadas que pertenecen a un contexto social más amplio y se basa en representaciones comunes de la realidad social.

Además, se usa aquí la noción de representación social como «la elaboración de un objeto social por una comunidad» o un "pensamiento de grupo" según el cual individuos se relacionan con un objeto (Dómic, 1999, pp. 6-12). Esta teoría, que volvió a introducir Moscovici en las ciencias sociales en los años 1980, vincula sociología y psicología y tiene así la ventaja de ser flexible y adaptable (Rateau \& Molinier, 2012). Esta noción entonces ayuda en el análisis de los procesos cognitivos involucrados en la movilización colectivas cuya importancia fue demostrada por un grupo significativo de sociólogos, mayormente de la teoría del frame analysis (Benford \& Snow, 2000). Además, permite dar una definición reducida de la noción de representación cultural. En este estudio, el concepto de "cultura» se usa en su significado antropológico amplio, es decir, un sistema dinámico y simbólico de representaciones, valores y rituales según los cuales se identifican y actúan individuos y grupos (Cuche, 2016). El análisis también se alinea con un «relativismo cultural científico" según el cual "cada sistema tiende a ser coherente y tener una cierta autonomía simbólica que le da su carácter singular original, y que no se puede analizar una característica cultural independientemente del sistema cultural del cual es parte, que le da un significado» (Cuche, 2016, p. 159).

Con entrevistas, observaciones en vivo, revisión en archivos y revista de textos académicos, fue posible trazar las líneas principales de la historia de esos grupos de NNAT y llegar a una comprensión tanto de las estructuras sociopolíticas como de 
las dinámicas subjetivas que llevaron organizaciones locales de NNAT a emerger como un movimiento social a escala nacional.

En total, se realizaron catorce entrevistas con actores diversos, entre los cuales se encontraban cuatro miembros y dos exmiembros de Taypinats, dos miembros y dos exmiembros del Consejo de Niños, Niñas y Adolescentes Trabajadores Organizados de El Alto (Connatsdea), un miembro de la organización NNAT de Potosí también coordinador de Unatsbo, y dos colaboradores. Se hicieron también entrevistas con dos psicólogos de la Fundación La Paz, donde nació Taypinats, y con una trabajadora del Ministerio de la Justicia. Así, se quiso reunir perspectivas de individuos de distintas edades que tuvieron un papel en la emergencia de Taypinats como parte de un movimiento social más amplio. También se llevó a cabo una entrevista con la directora de Act2gether Latinoamérica, ONG especializada en el derecho a la participación de los niños, nińas y adolescentes y que tiene conocimiento sobre la realidad local en términos de derechos, más que todo del derecho a la participación.

Además de contactos informales continuos con los miembros activos de Taypinats y trabajadores de la Fundación La Paz, el autor estuvo involucrado en un total de once observaciones, incluso en eventos estratégicos nacionales de Unatsbo, reuniones locales de Taypinats, una reunión organizada por el Ministerio de la Educación donde participaron varios NNAT de todo el país, un evento organizado conjuntamente por la Coalición Boliviana de Defensa de los Derechos de los Niños y la Redlamyc, y observaciones de varias horas con NNAT trabajando en las calles. Siendo la capital política del país, La Paz constituyó un lugar privilegiado de la observación etnográfica por dos razones principales. Primero, los eventos principales de las organizaciones NNAT, de la sociedad civil y del gobierno tuvieron lugar allá. Segundo, las movilizaciones de Unatsbo durante los eventos políticos mencionados, sucedieron en La Paz y Taypinats todavía tiene miembros que participaron en estas.

Cabe precisar aquí dos principales limitaciones de este trabajo. Primero, esta investigación no abarca la situación de todos los niños, niñas y adolescentes trabajadores de Bolivia. De hecho, no toma en cuenta cuatro categorías: (i) niños y niñas que trabajan en las áreas rurales, principalmente en la agricultura y la minería; (ii) niños y niñas que realizan trabajo doméstico; (iii) de manera más general, los y las que trabajan en la ciudad pero que no están en una organización NNAT, aunque la realidad de muchos puede ser similar, y (iv) niños y nińas que están en situación de calle y que, aunque muchos trabajan, viven una situación muy diferente de los NNAT de este estudio que vuelven a casa con sus familias al fin del día. No existen muchos datos disponibles para dar una idea del porcentaje de cada categoría en Bolivia. Estudios de 2012 estimaron que la mayoría de los niños, niñas y adolescentes bolivianos trabajan en la agricultura $(66,4 \%)$, mientras el $7,2 \%$ trabaja en actividades de hospitalidad y restauración, y 2,1\% en construcción. La industria de 
manufactura y las actividades de servicios representan ambas el 6,1\% de los niños, niñas y adolescentes trabajadores (Comunidad de Derechos Humanos, 2014, p. 18). Un estudio reciente, llevado a cabo en 2018 , calculó que, si la mayoría trabaja en áreas rurales, cerca del 35\% se encuentra en áreas urbanas (Comunidad de Derechos Humanos, 2014, p. 55).

Es por eso que este estudio quiere dar una idea de la situación y las representaciones de los nińas, niños y adolescentes trabajadores en áreas urbanas que laboran en la venta informal de productos y de comida (o formalmente en la manufactura para algunos), y que forman parte de una organización NNAT. Además, esta investigación proporciona información y análisis sobre la emergencia de una conciencia común en una organización NNAT y de la formación de un movimiento social de NNAT en el país. Por falta de tiempo y medios, el autor decidió enfocarse en una organización NNAT central en el movimiento nacional, pero, si es posible, este trabajo se deberá completar, comparar y criticar frente a otros estudios cualitativos hechos con organizaciones NNAT de los otros departamentos de Bolivia.

\section{Movilización de las organizaciones NNAT en Bolivia - Perspectiva HISTÓRICA Y ESTRUCTURA DE OPORTUNIDADES POLÍTICAS}

Bolivia no escapó a la tendencia que caracterizaba la mayoría de los países latinoamericanos en la segunda parte del siglo XX. En los ańos 1980, cuando emergieron por primera vez las estructuras de las cuales se formaron más tarde las organizaciones NNAT, la situación socioeconómica de Bolivia era trágica. Los dos dictadores Hugo Banzer (1971-1978) y el general Luis García Meza (1980-1981) «contrataron la deuda externa la más grande que Bolivia haya conocido» (González Ortega en González Ortega, 2017, p. 63). Cuando volvió la democracia en 1982, la tasa de inflación del país era de 27 000\% (García Linera, Chávez León \& Costas Monje, 2010, p. 66). Todo boliviano que vivió esa época o conoce la historia de su país diría que ese fue probablemente el momento más duro que Bolivia haya conocido.

Después de una cierta inestabilidad, el nuevo presidente elegido $-\mathrm{y}$ líder de la revolución de 1952 - Víctor Paz Estenssoro y su ministro del Planeamiento tenían una sola obsesión: controlar la inflación. Presionados por el Fondo Monetario Internacional (FMI), impusieron una serie de medidas draconianas con un toque de austeridad basado en el modelo neoliberal conocido como "Shock Therapy» (Silva, 2009). Estas medidas se intensificaron en la segunda mitad de los años 1980, y tuvieron inevitablemente un efecto desastroso sobre la población. La pobreza generalizada y la ausencia de medidas sociales para responder a las necesidades básicas de la mayoría de los bolivianos culminaron en la Marcha por la Vida, en 1986. Esta reunió a trabajadores de todos los sectores, incluso del sector informal 
(que todavía es mayoritario en el país), a manifestar en las grandes ciudades contra las reformas neoliberales y el corte a los presupuestos sociales (Calderón, 1995; Silva, 2009; Gonzalez Ortega, 2017). Como recientemente escribió el historiador y expresidente Carlos Mesa, «tras la hecatombe económica, estaba claro que la estabilización tenía inevitablemente que ir acompañada de exclusión, desempleo e informalidad económica» (Mesa Gisbert en González Ortega, 2017, p. 273). En este contexto, millones de personas migraron a las ciudades en busca de trabajo y otros medios para sobrevivir. Bien sea por necesidad económica, factores culturales o la imbricación de los dos, una gran cantidad de nińos salieron a la calle a trabajar, con su familia o solos, vendiendo todo tipo de productos y proveyendo servicios.

Al mismo tiempo, el retorno de la democracia permitió la formación de asociaciones y la entrada de ayuda internacional a Bolivia, hasta entonces respectivamente perseguidas y prohibida por las dictaduras consecutivas. Entre 1980 y 1993, "época dorada» de las ONG en la región latinoamericana, se multiplicó por cinco el número de esas organizaciones en Bolivia (Dumoulin, 2000), con más de quinientos a través del país en el medio de los años 1990 (Silva, 2009, p. 109). Además de permitir el desarrollo de varios proyectos con fines sociales, esas organizaciones trajeron con ellas el lenguaje omnipresente de los derechos humanos y particularmente, después de la Convención Internacional sobre los Derechos del Niño $(\mathrm{CDN})$. Asimismo, emergió progresivamente un discurso enfocado en los niños, niñas y adolescentes como sujetos de derechos y dignidad, entendido hoy como el paradigma de la protección integral. Eso inició un mayor cambio en la manera de ver los niños en el espacio político dominante, porque lo que predominaba hasta entonces era el paradigma tutelar. Este correspondía a una visión ideal del nińo, que era objeto a educar, y consideraba a los nińos, niñas y adolescentes en la calle como un problema social a erradicar (Morais de Ramírez, 2016; Pochetti, 2018).

En ese contexto se desarrolló el primer proyecto colaborativo dirigido a nińos, niñas y adolescentes vulnerables de La Paz. Un grupo de especialistas de la Fundación La Paz, incluyendo J. Dómic, con el apoyo de algunas ONG (la ONG Cáritas Suecia como socio principal), crearon en 1986 un proyecto original e inclusivo con el objetivo de ayudar a los niños trabajadores en las calles de la ciudad. Después de tres años de investigación y preparación, se dieron cuenta de que los niños y adolescentes en La Paz se enfrentaban a diversas realidades. Así, un mayor proyecto, llamado Sarantañani, vio la luz. Estaba formado de tres subproyectos destinados respectivamente a (i) nińos y adolescentes que trabajan y viven solos en la calle, llamado Sarantañani Calle; (ii) niños y adolescentes que trabajan en la calle y vuelven a casa con su familia en la noche, Sarantañani Trabajador, y (iii) niñas y adolescentes de la calle, llamado Okarina. En los últimos treinta ańos, Sarantañani dio soporte moral, infraestructuras técnicas y formaciones en competencias necesarias 
a numerosos NNAT. De los tres proyectos, el que impulsó la creación de Taypinats fue el Sarantañani Trabajador, que duró hasta 2017.

El proyecto Sarantañani Trabajador sin duda fue central en reunir varios NNAT y darles las condiciones materiales para organizarse. En este espacio de socialización, $\mathrm{y}$ tras acciones grupales, los NNAT llegaron a la conciencia de existir como un grupo social —y luego político-, enfrentado a los mismos problemas y desafíos. En los años 1990, nació Taypinats, la organización de niñas, niños y adolescentes trabajadores de La Paz, que todavía tiene su oficina en la Fundación La Paz (ver fotografía 1).

Fotografía 1. Fundación La Paz, donde nació la organización Taypinats. Su oficina se sitúa en el primer piso. Fotografía tomada el 17 de julio 2018.

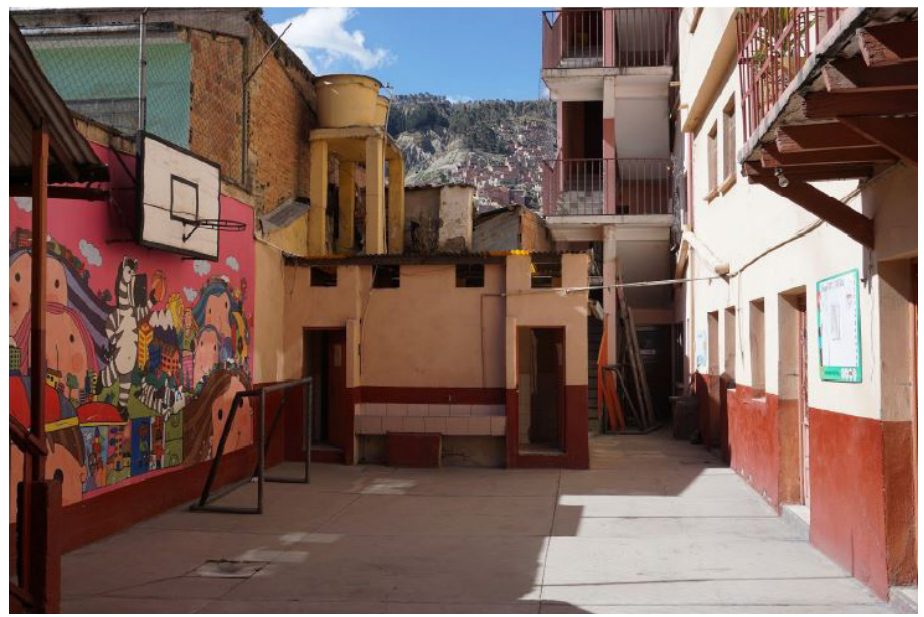

Mathieu explica que, según Tarrow y Tilly, la estructura de oportunidad política (structure of political opportunity, SPO) se basa en seis elementos, incluyendo «la apertura a nuevos actores», «la disponibilidad de aliados influyentes» $\mathrm{y}$ «la capacidad del régimen de facilitar o represar las expresiones colectivas de las demandas» (Mathieu en Filleule, Mathieu \& Péchu, 2009, pp. 530-540). Además, «esta estructura se considera "abierta" cuando el contexto es favorable para un movimiento social, especialmente cuando el sistema político muestra receptividad a sus demandas; sin embargo, esta estructura será "cerrada" cuando el sistema político rechaza de manera intransigente la negociación, o aún escoge la opción de la represión» (Lilian en Agrikoliansky et al., 2010, p. 42). En el caso de Unatsbo, se puede suponer que la organización nació de la agregación de varias organizaciones locales de NNAT que siguieron más o menos la misma lógica que Taypinats en un contexto de apertura de la SPO que no solo dio espacio a la ayuda social, sino también a la contestación social y la movilización colectiva. Además, la llegada 
al poder de Evo Morales, líder de un movimiento social nacional y ex NNAT, creó condiciones favorables para la acción colectiva de los NNAT y su implicación más tarde en la escritura de la Constitución del Estado Plurinacional de Bolivia y del Nuevo CNNA.

Sin embargo, estos procesos históricos y los cambios en la estructura política por sí solos no permiten entender cómo los NNAT tomaron conciencia de pertenecer a un grupo y decidieron entrar en conflicto con las autoridades. En otras palabras, en nuestro caso, mientras estos elementos explican las bases de la creación de la organización Taypinats, no explican su participación en un movimiento social de amplitud nacional. Por eso, se necesita adoptar un enfoque más cognitivo que implica la psicología social y permita poner la luz sobre la significación que los actores dan a su situación (Contamin, en Agrikoliansky et al., 2010). Aquí, la metodología etnográfica parece esencial, y como ya lo notaron McAdam, Tarrow y Tilly, «[e]n comparación con otras técnicas, investigadores han hecho un uso mínimo del método etnográfico para examinar las dinámicas de las protestas, a pesar del hecho que la fuerza de este método se encuentra en la atención que da a estos procesos» (McAdam, Tarrow \& Tilly, 2008, p. 310).

\section{¿Quiénes son los NNAT? Perfiles de los entreVistados E IMPORTanCia DE LA AYUDA}

Nadia tiene dieciséis ańos y trabaja desde los nueve en las calles de La Paz. Empezó a vender con su madre, para "ayudarla», dice. Al inicio, trabajaba poco, hasta que se fue a otra ciudad para integrar una escuela militar. Sin embargo, la pérdida de su papá la obligó a volver a La Paz para, esta vez, trabajar de manera regular y apoyar económicamente al hogar. Ayudó a su tía en su taller e hizo pequeños trabajos informales, más que todo con la familia. Hoy, además de ser miembro activo de Taypinats en el ámbito local y coordinadora de Unatsbo a escala nacional, Nadia trabaja cada mańana de las seis a las doce en un mercado en el centro de La Paz, practica natación varias veces por semana, estudia y sigue clases particulares de inglés que paga con lo que gana en el mercado. Aunque cada NNAT tiene su experiencia específica, el perfil de Nadia es representativo de un patrón más general que caracteriza a numeroso NNAT, es decir, un individuo de menos de dieciocho años que estudia, trabaja para apoyar a la familia y puede ganar dinero; viene de una familia con una situación socioeconómica vulnerable y se involucra en una organización NNAT.

Los miembros y exmiembros de Taypinats entrevistados fueron Roberto Carlos, diez años, vendedor en las calles de la zona sur de La Paz; Juan, dieciséis años, que trabaja en una industria de fabricación de zapatos con su padre; Nadia, dieciséis 
años, que trabaja con su tía en un mercado en la calle en el centro de La Paz; Pedro, dieciséis años, vendedor en las calles del centro de La Paz y músico; Lizet, diecinueve años, ex NNAT y colaboradora, y Héctor, veintiún años, ex NNAT y colaborador. Los miembros del Connatsdea entrevistados fueron Simón, catorce años, vendedor en las calles de El Alto; Raúl, dieciséis años, que trabaja en una carnecería; Álvaro, dieciséis años, que trabaja en una industria de fabricación de disfraces; Gimena y Marcelo, veinte y veintiún años, exmiembros y colaboradores del Connatsdea. También se tuvo la oportunidad de entrevistar a Estéfani, dieciséis años, vendedora en las calles de Potosí, coordinadora de Unatsbo y miembro del del Consejo de Niños, Niñas y Adolescentes Trabajadores Organizados de Potosí (Connat'sop) desde sus nueve ańos.

Cabe notar que, aparte de Roberto Carlos, la mayoría de los NNAT tiene alrededor de quince años. Esto se explica, quizás, por dos razones. Primero, el presupuesto de Sarantańani se acabó en 2017 y se basa hoy únicamente en una ayuda financiera de la Fundación La Paz. Así, sus actividades son significativamente menos intensas y Taypinats no tiene dinero como para comprar productos de primera necesidad que ayudaban y atraían a numerosos niños, niñas y adolescentes trabajadores. Vinculado con esto, la segunda razón se debe al hecho de que los NNAT entre catorce y quince años - generalmente miembros desde hace varios años- participaron en las confrontaciones con las autoridades de 2013-2014 y, para algunos, de 2008, y desarrollaron un compromiso fuerte y durable.

La mayoría de los NNAT trabajan como vendedores en las calles o apoyando a sus padres, es decir, de manera informal. De hecho, una vasta mayoría de niños, niñas y adolescentes trabajan en el sector informal, lógica que se encuentra en casi toda la región latinoamericana y, aunque su amplitud disminuyó, se observa desde hace varias décadas (Salazar, 1998; Liebel, 2004; Bourdillon 2006; Cussiánovich, 2010; Cavagnoud, 2014; Morsolin, 2014 y 2017). Por ejemplo, Roberto Carlos vende en las calles, solo o con su madre, birretes y otros accesorios para el cabello (ver fotografía 2). Otros, como Lizet, Estéfani o Simón, han llevado diversos trabajos informales, generalmente en un taller familiar, en un negocio de comida o vendiendo productos básicos en las calles de la ciudad. Marcelo empezó a trabajar a los nueve años en el sector de la construcción con su padre, para «ayudarlo». Algunos, como Juan, Álvaro y Raúl trabajan formalmente para alguien, en una empresa manufactura. En el caso de Juan, su padre, con quien empezó a trabajar en la misma manufactura, les enseñó a hacer zapatos. Raúl empezó trabajando en un autobús y aprendió poco a poco a manejar. Luego, dejó el transporte para trabajar en restaurantes, y hoy vende carne en una tienda. En el caso de Álvaro, fueron sus amigos quienes lo llevaron a un taller de fabricación de ropa para la diablada —un baile popular boliviano- cuyo dueño lo contrató y le enseñó técnicas de costura. 
Hoy, Álvaro dice que no trabaja más, pero que «ayuda» a su padre a coser pelotas que van a vender después.

Algo que todos los NNAT entrevistados tienen en común son las razones por las cuales empezaron a trabajar. Estas oscilan entre el deseo de (i) participar en la economía de familia y su sustento, y (ii) de ganar su dinero para aliviar un peso económico sobre los padres y poder pagarse cosas de recreo y de estudio. Casi todos mencionaron que empezaron a trabajar alrededor de los diez ańos - a veces mucho antes-, al lado de un miembro de la familia. Dos dijeron que empezaron solos, impulsados por amigos que ya trabajaban. No obstante, cuando se pregunta: “¿Por qué empezaste a trabajar?», la respuesta es, invariablemente: «Para ayudar a mi mamá/papá». Esta idea de ayuda —o de apoyo — a la familia está profundamente arraigada en el pensamiento de los niños y adolescentes entrevistados. Lo que puede cambiar es la presencia o ausencia de necesidad, aunque la segunda es mucho menos frecuente. Algunos de los NNAT entrevistados respondieron de la siguiente manera:

- Sí, es que trabajaba desde los ocho años, ayudaba a mi madre a vender bolsillos con cosas para parejas.

- Para ser honesta, yo escogi trabajar, mh, antes de los doce años. Empecé a trabajar desde pequeña, siempre iba a vender, digamos, siempre atraía mi atención.

- Sí, estoy trabajando, estoy ayudando a mis padres.

- Con el dinero con el que trabajo, una parte lo guardo para mis estudios para la universidad, y otra parte va a mis útiles: comprar cuadernos, esas cosas.

- Mh... [uso mi dinero] mayormente para mis gastos personales y algunas veces para ayudar a mi mamá [...] [compro] comida, a veces ropa a salir de paseo. Y para el colegio, alguno y otro libro.

Para algunos NNAT, la voluntad de ayudar acompañó la toma de conciencia de una necesidad económica urgente. Para otros, resultó del único deseo de apoyar a la familia y aprender.

Como analizaron los pocos académicos que trabajaron con NNAT en el área andina, más allá de tener una noción positiva del trabajo, los NNAT lo consideran como un factor importante de su desarrollo (Dómic, 1999; Liebel, 2003 y 2004; Bourdillon, 2010; Cussiánovich, 2010; Okyere, 2012; Cavagnoud, 2014; Morsolin, 2017). De hecho, los NNAT ven su trabajo de manera generalmente positiva, con variaciones que dependen de la situación del individuo. Existe un dicho en el idioma aimara que varios NNAT mencionaron y que significa: «No seas ladrón, no seas mentiroso, no seas flojo». Los NNAT entrevistados representan la segunda o tercera generación desde el éxodo rural que trajo millones de bolivianos desde el campo hasta la ciudad. La mayoría de los niños y adolescentes son mestizos y tienen 
poco —o ningún — vínculo con el campo. Por lo tanto, en el marco de esta investigación es imposible decir en qué medida la cultura aimara, presente mayormente en zonas rurales, se encuentra en la ciudad. Tal análisis necesitaría un trabajo histórico y antropológico más específico. De cualquier manera, el valor negativo asociado al «ser flojo» es fuerte y todos los NNAT rechazan esta posibilidad y ven el trabajo como parte integral de su desarrollo.

- Me da energía. Ojalá pueda trabajar hasta los treinta ańos. Ah, sí, me gusta vender porque hay momentos que, digamos, me dan felicidad.

- Mh, no, [mis padres] no querían que trabajara. No querían, pero al mismo tiempo, estaba lleno de este sentimiento, esta necesidad, porque no teníamos que comer y bueno, esto me dio, como puedo decir... tenía esta voluntad de trabajar para ayudar también, no quedarme estático o, como podría decir de otra manera, flojo.

Hablar con varios NNAT muestra, entonces, un patrón en el cual el trabajo no solo está —y no siempre — vinculado con la necesidad, sino también con el apoyo a la familia, con el desarrollo individual. Claramente, no se puede destacar la necesidad económica. Bolivia es el país más pobre de América del Sur y la situación económica de numerosas familias contribuye a llevar a los niños a practicar una actividad económica. Sin embargo, el peso del factor socioeconómico se debe matizar con el del factor cultural, es decir, de las representaciones de las familias y de los NNAT

Fotografía 2. Roberto Carlos saliendo de la escuela y vendiendo birretes y otros accesorios con su madre en el barrio de San Miguel en la Zona Sur de La Paz. Fotografía tomada durante una observación de terreno el 7 de julio 2018.

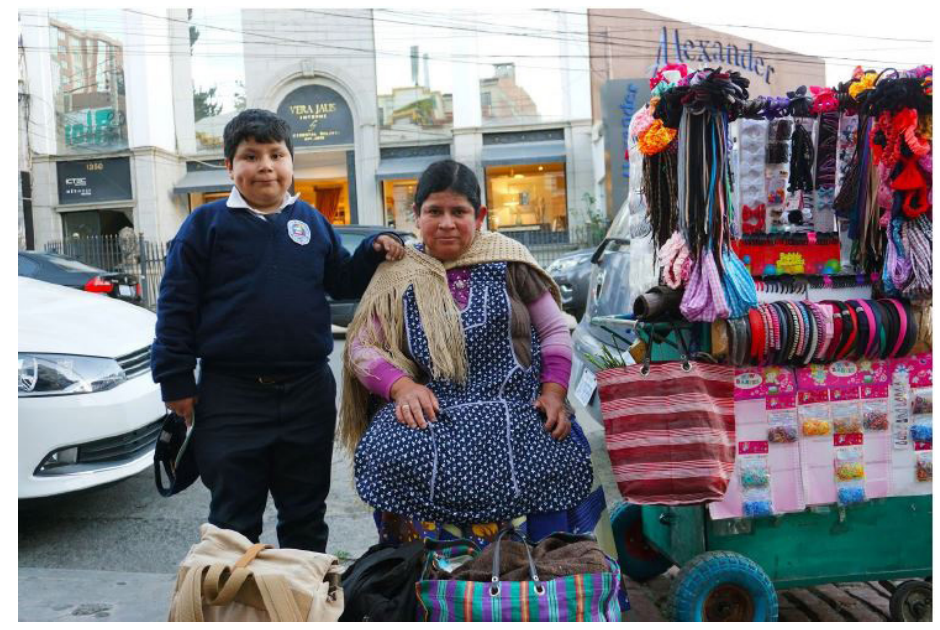


sobre el trabajo y la infancia. Estas entrevistas y estudios previos con NNAT latinoamericanos muestran que, de manera general, el trabajo se entiende como contrario de explotación y como una actividad positiva de continuidad de la familia y aceptación por la comunidad por no ser flojo. Aunque está más que todo vinculado a la necesidad, los nińos y adolescentes reconocen que, en la legalidad o no, tienen que trabajar porque quieren apoyar a su familia, pagarse sus necesidades básicas y el material para poder estudiar.

\section{«SOY NNAT!» - Conciencia COlectiva Y MOVILIZaCión}

Una cuestión central en esta investigación es entender el proceso de (i) identificación a la identidad NNAT de estos niños, niñas y adolescentes, y (ii) adquisición de una conciencia política que permitió la emergencia del movimiento Unatsbo.

Mientras tenía presupuesto, Sarantańani fue un proyecto inclusivo y multidimensional mezclando acciones en las calles y educación tanto práctica como académica. Isbel, trabajadora de la Fundación La Paz involucrada en el proyecto durante cerca de diez años, cuenta que los niños, niñas y adolescentes iban a las calles para hablar con los trabajadores y trabajadoras de menos de dieciocho años que no conocían el proyecto, dándoles folletos y comida. En la fundación, algunos trabajadores y adolescentes daban - y siguen dando- clases sobre la historia de Bolivia y los derechos de los niños. Antes, varios especialistas también enseñaban competencias prácticas, como cocinar o coser. Había también una cafetería con comida gratis para todos, pero los niños, niñas y adolescentes instauraron una participación simbólica de un boliviano por una comida. El dinero iba a las actividades de grupo. Estas actividades y dinámicas se encuentran también hoy, pero con una amplitud bastante reducida debido a la falta de presupuesto.

La mayoría de los niños, niñas y adolescentes empezaron a ir a la fundación porque un amigo que estaba involucrado en el proyecto les dijo que había un lugar con clases, amigos y comida. En el caso de Lizet, un grupo de nińos y nińas se le acercaron con un folleto contando lo que estaban haciendo en un lugar donde había muchas y muchos que, como ella, trabajan. Juan y Pedro se apasionan por la música. Cuentan que fueron a la fundación por la primera vez, respectivamente en 2013 y 2016, porque había clases para enseñar a tocar un instrumento, salas disponibles para ensayar y, por qué no, formar una banda. Según trabajadores de la fundación y ex NNAT, había cerca de doscientos niños, niñas y adolescentes trabajadores de La Paz y El Alto participando en algún tipo de actividad del proyecto Sarantañani en el año 2007. La mayoría participaron en las manifestaciones de 2008 y, luego, de 2013-2014.

Participar en acciones en las calles, o simplemente venir una hora para comer o dormir en la fundación, marca un hito en la vida de muchos niños, niñas y adolescentes 
trabajadores. Toman conciencia del hecho de que no están solos y que hay otros niños que viven aproximadamente la misma realidad. Roberto Carlos, por ejemplo, cuenta que le gusta ir con Taypinats "porque hay niños que venden también, hay muchos amiguitos», y luego admitió que "la comida era deliciosa». Juan comparte que un amigo le llevó a la fundación, donde «me gustaba conocer a gente que trabaja, yo pensaba que era solo entre pocos otros». Gimena, hoy colaboradora, cuenta:

Hay muchas experiencias de vida, algunas tristes, que siempre se pueden contar. Compartimos, cuando nos veíamos siempre compartíamos historias de cómo era nuestro trabajo o lo que hacíamos antes de hacer parte de la Unatsbo. Era muy agradable conocerlos, reír, jugar con ellos. Eso era, eso es, como ser parte de otra familia.

Según lo anterior, aparece que, si los niños, niñas y adolescentes tienen su primer contacto con la fundación por razones materiales, de necesidad o de recreo, deciden volver porque conocen a otros en las mismas condiciones, hacen amigos, crean vínculos y sienten que forman parte de un grupo. Es decir, se sienten menos solos y más entendidos, mientras que, en su entorno cotidiano, compañeros de escuela los pueden juzgar por ser trabajadores y probablemente "pobres». Para algunos niños, niñas y adolescentes, a través de clases sobre la historia del país y los derechos humanos dadas por colaboradores y NNAT emerge un interés político. De esta forma, se desarrolla la conciencia de pertenecer a un grupo con individuos con una situación similar y una lucha común.

Desde su creación, un elemento fundamental del proyecto Sarantañani es el de dar una voz y promover su autorganización. Es decir que, como ya se mencionó, la Fundación La Paz ha proporcionado medios materiales y conocimiento, pero la mayoría de las actividades dependen de la iniciativa de los NNAT y ellos mismos las lideran. Este principio se cristalizó en los años 1990, cuando Taypinats se identificó como una organización independiente de NNAT y el principio de participación se inscribió en la carta de la organización. Así, mientras algunas actividades educativas y las comidas eran la responsabilidad de la fundación bajo Sarantañani, un grupo de niños, niñas y adolescentes tomaron el liderazgo y participaron en la creación de Taypinats como organización independiente.

Varias observaciones en eventos locales y nacionales, junto con respuestas de entrevistados, mostraron que, desde el inicio, las organizaciones NNAT funcionan de manera estructurada, donde cada NNAT tiene una voz de la misma importancia y donde participan colaboradores que no tienen poder de decisión. Generalmente, un colaborador hace una presentación de la situación actual vinculada con el contexto boliviano y los derechos de los niños. Menciona después el objetivo de la reunión para la organización y se ocupa de establecer orientaciones claras. Los adultos no intervienen más y dejan a los miembros NNAT debatir y discutir sobre 
los temas, y luego decidir del planeamiento de las actividades de la organización NNAT en los ámbitos local, o nacional para Unatsbo. Además, cuando se debe tomar decisiones importantes, se pide a los colaboradores que dejen el espacio de reunión para no influenciar a los NNAT.

Las entrevistas muestran que, compartiendo esos momentos como una «reunión política» o momentos más informales, los niños, niñas y adolescentes adquieren el sentido de formar parte de un grupo social más amplio que los apoya y a quienes deben apoyar. En sus formas más extremas, este sentido implica referencias a la familia, como mencionó Gimena o como también comenta Nadia, que cuenta:

Me atraen mucho en este sentido las clases, la gente que he conocido. Tengo amigos allá también, entonces me quedé. $\mathrm{Al}$ inicio, era como conocer a amigos y tomar clases y aprender más, así, pero después de un tiempo, ya un año después, me he involucrado más en la organización, porque teníamos reuniones con el Ministerio de la Educación, ;tuvimos una reunión con el presidente! Socializamos este tema de los niños trabajadores

Es en estos espacios de integración, de intercambio, de aprendizaje, de participación y de debates donde los NNAT consolidan su identidad y desarrollan una conciencia de grupo y, en ciertos casos, política. Asimismo, estos individuos acaban por identificarse como NNAT. Es interesante notar que NNAT es plural y se usa de esta forma para calificar tanto una persona sola como un grupo de individuos, como si hubiera sido desmaterializado para solo referirse a la identidad, es decir, un ser NNAT. Además, se observa que esas organizaciones insisten en el uso del femenino primero y el masculino luego, traduciendo la importancia dada a la igualdad de género y, de manera general, la diversidad. El esquema siguiente resume el análisis proporcionado en esta sección:

Figura 1. Esquema analítico de la configuración identitaria NNAT

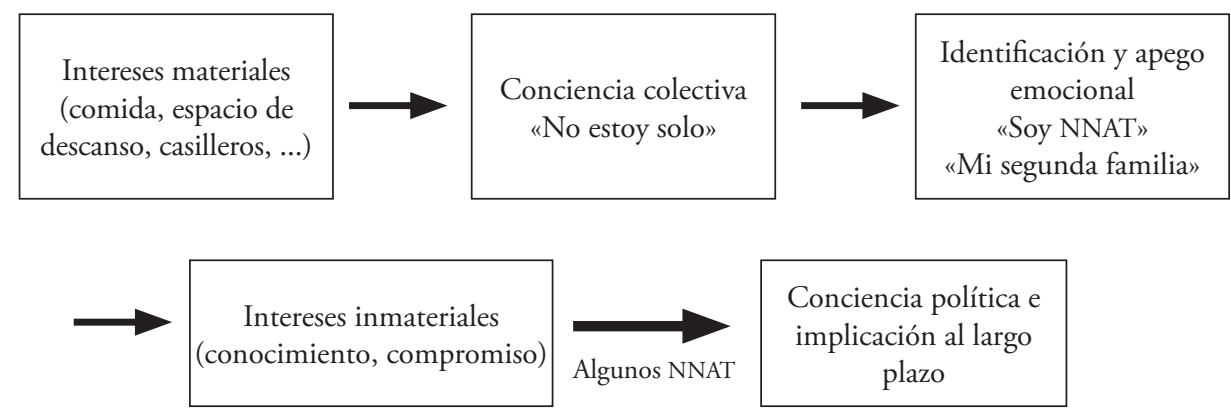

En conclusión, tras los procesos expuestos, emergió una identidad colectiva, un ser NNAT, basada en representaciones sociales comunes y una manera similar de 
percibir su realidad. Según esas representaciones, la niñez y el trabajo no son necesariamente dos mundos separados, y el segundo tiene una dimensión no solo de ayuda económica a la familia, sino también de desarrollo personal. En este sentido, tampoco se encuentra contrario a la escuela. Así, construyeron «una base lógica para comprometerse juntos en la acción colectiva de mejoramiento, incluso en la construcción de una acción colectiva apropiada» (Benford y Snow, 2000, p. 617). Taypinats ha organizado, hasta ahora, su propia lucha a escala local, y se unió a otros movimientos para formar en 2003 la Unión de Niñas, Niños y Adolescentes Trabajadores de Bolivia - Unatsbo (Morsolin, 2017), llevando acciones nacionales que han reforzado la identidad NNAT.

La lógica de ayuda de Sarantañani, impulsada por dinámicas históricas mencionadas antes, no desapareció. Sin embargo, algunos de los NNAT empezaron a seguir clases sobre los derechos de los nińos en la fundación, transmitir su conocimiento a otros niños y tomar del liderazgo de la organización Taypinats. Cuando se abrió la estructura política con la llegada de Evo Morales al poder, pudieron intensificar su lucha y participaron en manifestaciones y eventos mayores en la historia política del país donde se reforzó esa identidad colectiva. Juntos colaboraron con el Estado varias veces, pero también se enfrentaron a él. Su conexión con organizaciones internacionales y su historia anclada a la lucha social dieron a esas organizaciones de NNAT una dimensión fuertemente política, con un lenguaje de derechos humanos apremiante y central en sus reivindicaciones. Este lenguaje es también la expresión de la dimensión transnacional de los movimientos NNAT, nivel en el cual encuentran la mayor oposición a sus demandas, principalmente a través del trabajo de organizaciones como la OIT que participan en la construcción de paradigmas dominantes sobre el trabajo infantil.

\section{RELATIVIDAD DE LAS REPRESENTACIONES SOBRE LA INFANCIA. EL NIÑO TRABAJADOR Y PROTAGONISTA Y CRÍTICA DE LA VISIÓN OCCIDENTAL DEL SER NIÑO}

Mientras que en Europa y América del Norte no es común observar a niños, niñas y adolescentes en el espacio social y político, los NNAT bolivianos demuestran otra realidad. En 2016, la OIT estimaba que, de los 151622 millones de niñas, niños y adolescentes de cinco a diecisiete años involucrados en el trabajo infantil en el mundo, unos 10461 millones están en América Latina y el Caribe, lo que representa el 7,3\% de la población de niñas, niños y adolescentes de la región (Alliance 8.7, 2017, pp. 2-3). Pocos datos existen sobre Bolivia, pero la encuesta de niñas, niños y adolescentes que realizan una actividad laboral (ENNA) del INE calculó que, de los tres millones de niñas, niños y adolescentes en el país, cerca de 739000 están involucrados en alguna actividad laboral. De estos, 393000 están en el trabajo infantil según las definiciones de la OIT. 
Desde que Bolivia ratificó en 1997 el Convenio 138 de la OIT sobre la edad mínima, se fijó en catorce años la edad mínima para trabajar, lo que sitúa a cualquier niño, niña o adolescente de menos de catorce años en actividad laboral en situación de ilegalidad. Sin embargo, los datos de la Alliance 8.7, una alianza de organizaciones gubernamentales y de la sociedad civil que busca acabar con el trabajo forzado, la esclavitud moderna, el tráfico de humanos y el trabajo infantil, muestran que una gran parte (37\%) de los niños en el trabajo infantil en las Américas tiene entre cinco y once años (Alliance 8.7, 2017, p. 4). En Bolivia, «la población de nińos entre 5 y 13 ańos en actividad laboral corresponde a $491000,57,80 \%$ del total de niños trabajadores» (Coalición Boliviana por los Derechos de la Niñas, Nińos y Adolescentes, 2018, p. 56).

Los NNAT consideran que el convenio sobre la edad mínima no es representativo de su realidad y que el establecimiento de una edad mínima es arbitrario y no se puede aplicar en el contexto boliviano de la misma manera que en países occidentales, donde la visión de la nińez, las prácticas culturales y las condiciones socioeconómicas son muy diferentes. Así, bajar —o suprimir — la edad mínima legal para trabajar en Bolivia ha constituido una demanda central en la lucha de Unatsbo. Esta demanda común a todas las organizaciones NNAT los llevó a una respuesta bastante violenta hacia el Estado en 2013, cuando se percataron de que las autoridades no los habían escuchado y habían dejado las normas fijadas por la OIT. Siguiendo una mediatización sin precedentes en el ámbito internacional y, paradójicamente, debido a ciertas presiones internacionales, el gobierno de Evo Morales abrió de nuevo las negociaciones con los NNAT, consiguiendo finalmente bajar la edad mínima a los nueve años y participar en la modificación de algunos artículos. Consideran hoy que esto fue la mayor victoria de la historia de la Unatsbo.

Además, tanto los NNAT como los académicos critican la falta de claridad y el carácter también arbitrario de las definiciones de la OIT que se aplican como normas incontestables a los países. Jorge Dómic proporcionó una explicación que refleja este punto de manera clara:

[...] no hay un nińo universal y sobre esto tenemos que quedar claros. En los Andes, el niño es distinto. Es un niño que socializa al través del trabajo y otros elementos, y en términos de representación social, el trabajo es también distinto. La OIT no tiene un concepto, lo que tiene la OIT es una definición operativa del trabajo vinculada a una concepción general, obviamente muy distinta. La OIT no ha hecho en ningún momento una diferencia entre lo que es la explotación y lo que es el trabajo; pero esto no es el mismo para nosotros - el trabajo es una cosa y la explotación es otra (entrevista personal).

Los NNAT hacen una distinción clara entre las nociones de trabajo y de explotación y consideran que tratar de «erradicar» el trabajo de los niños, niñas y adolescentes no solo es imposible sino también discriminatorio. Nadia nota, por 
ejemplo, que «[h]ay explotación, pero desde el Código 548, se ha reducido esa parte. Aunque la OIT está en contra de nosotros, dicen no, que no... Que niños no deberían trabajar porque nos hacemos mal ver en Bolivia, pero la realidad no es así, es otra. En cada familia, cada niño nace con otra realidad».

No obstante, cabe notar que la organización internacional ha hecho progresos significativos en términos de adoptar una definición más amplia del trabajo de los niños, niñas y adolescentes. Aunque la distinción entre el trabajo y el trabajo forzado se haya ignorado durante mucho tiempo, establecieron recientemente que «no todos los trabajos hechos por niños se deben clasificar como child labour que se debe erradicar. La participación en actividades laborales de niños y adolescentes que no afecta su salud o desarrollo personal o interfiere con su educación, es generalmente visto como algo positivo». El problema se sitúa entonces en dos elementos principales. Primero, el concepto de child labour se refiere a la explotación laboral e a todos los niños, niñas y adolescentes involucrados en una actividad laboral bajo la edad mínima fijada por la organización. Vinculado con esto, el segundo elemento se refiere a la falta de claridad y la interpretación de las definiciones y, más que todo, a la falta de participación de los interesados — aquí los NNAT — para aplicarlas a las realidades de los contextos locales.

De hecho, la segunda demanda fundamental de la lucha de Unatsbo es el respeto del derecho a la participación de los niños, niñas y adolescentes. Además del convenio sobre la edad mínima, Bolivia también firmó en 2003 el 189 Convenio sobre las peores formas de trabajo infantil y la Convención sobre los Derechos del Niño (CDN) de 1989. Los NNAT también se sirven de documentos de derechos humanos como la CDN para denunciar el hecho de que el Estado boliviano y organizaciones internacionales como la OIT no respetan su derecho a la participación ni su contexto local. Así, por ejemplo, en las manifestaciones y los folletos de organizaciones, se puede leer casi siempre el eslogan "por un trabajo digno» (ver fotografía 3), lo que refleja el concepto de dignidad presente en las convenciones internacionales de derechos humanos. Cuando pregunté a Estéfani por la importancia que tiene para ella la participación y su experiencia de lucha, me respondió: «Nosotros, avanzamos mucho en Bolivia, con la Unatsbo avanzamos mucho, porque nos hicimos respetar como organización de niños trabajadores, entonces nos dan muchas posibilidades, posibilidades que ni autoridad ni nadie se puede burlar de nosotros, y sí, tenemos que tomarnos en cuenta».

El terreno realizado durante esta investigación y estudios previos muestran que los NNAT se ven como miembros activos de la sociedad, en la cual se deben considerar como actores y sujetos de derecho. Lamoureux, una socióloga que parte de un reciente movimiento estadounidense enfocado en los derechos humanos como contextualizados y dinámicos, escribe que «la lucha por los derechos cuestiona 
las fundaciones de lo que es justo e injusto en una sociedad y obliga las autoridades a justificarse ellas mismas y sus acciones. Podemos entonces decir que los individuos que luchan por sus derechos actúan políticamente pidiendo la legitimidad de las fundaciones del poder en una colectividad y que, en este sentido, son sujetos políticos» (Lamoureux en Truchon \& Saillant, 2013). Esto supone dos elementos fundamentales. Primero, los derechos humanos tienen una «vida social» (Truchon \& Saillant, 2013), se expresan y se definen diferentemente en contextos diferentes. Por lo tanto, no se pueden imponer desde "arriba», sin tomar en cuenta la situación social, económica y cultural de una región, país o localidad. Segundo, el uso de los derechos como lenguaje y herramienta, y no solo un fin en sí, significa que los NNAT son sujetos políticos y así pues son actores «capaces» en su comunidad y en la sociedad boliviana.

\section{Fotografía 3. Folleto del año 2015 del proyecto Sarantańani Trabajador}

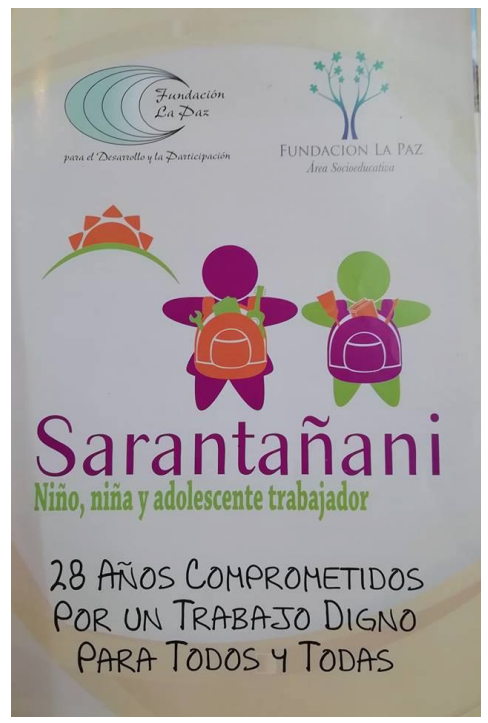

Varios autores, empezando con la antropóloga Margaret Mead (1926), pusieron el énfasis en el hecho de que hay varias maneras de ser y de que "la diversidad en la manera de considerar la infancia y vivirla refleja el hecho de ser nińo como una construcción social a través de series de imágenes heterogéneas, representaciones y códigos que son diferentes de un solo desarrollo biológico" (Cavagnoud, de Sumerian \& La Riva Gonzales, 2013, p. 325). Un grupo significativo de académicos también mostraron que las organizaciones internacionales, cuyas normas tienden a imponerse en los niveles nacionales y locales, reflejan una visión estandarizada de la niñez que encuentra sus raíces en la historia de los países occidentales. Esta visión 
se quedó como un ideal a seguir, sin tomar en cuenta criticismos y variaciones culturales (Invernizzi, 2003; Liebel 2004; Bonnet \& Schlemmer, 2009; Bourdillon, 2010; Cussianovich, 2010). Holzscheiter (2013) explica finalmente que está lógica también se encuentra en organizaciones de la sociedad civil «que si sitúan entre una herencia histórica de un proteccionismo altruista (organizaciones caritativas del norte) e intereses personales (uniones de obreros) ${ }^{1}$ por un lado, y el reconocimiento y promoción de los derechos humanos, de la agency y de empoderamiento de los niños por otro lado» (p. 208). Como mencionó Lizet refiriéndose a esas organizaciones, «[...] nos ignoran, es muy raro que defiendan a los trabajadores, pero no a los NNAT. Somos también trabajadores, también trabajamos»².

Alejandro Cussianovich director del Instituto de Formación de Educadores de Jóvenes, Adolescentes y Niños Trabajadores (Ifejant), que trabajó una gran parte de sus vidas con NNAT peruanos, fue el primero en desarrollar el concepto de "protagonismo" de los NNAT. En su Ensayo sobre la Infancia, define el "protagonismo» como un "paradigma conceptual» profundamente vinculado con el derecho a la participación, según el cual «la participación es una cuestión de poder, ejercicio del poder, e importante notar, es parte de la red social, la relación entre la sociedad civil, el Estado, actores sociales»(Cussiánovich, 2010, p. 60). El protagonista es, entonces, un individuo que ocupa el espacio social y político en calidad de actor, y no solo como recipiente. En este sentido, los NNAT — no solo en Bolivia, sino también en la mayor parte de América Latina (Morsolin, 2009)_ contribuyen a la construcción de la sociedad.

\section{ConClusión}

Esta investigación arrojó diferentes procesos que llevaron a los NNAT bolivianos a organizarse en los ámbitos local y nacional y a movilizarse colectivamente para contestar autoridades y defender sus intereses. El análisis mostró primero que la apertura política y la llegada de ayuda internacional a una Bolivia devastada por décadas de dictadura y de austeridad económica, permitieron la implementación de proyectos locales con la ayuda de algunas ONG internacionales para ayudar a los más vulnerables. Eso fue el caso de la Fundación La Paz, que desarrolló el proyecto Sarantañani Trabajador, que ha apoyado materialmente y moralmente cientos de

\footnotetext{
1 En sus entrevistas, Jorge Dómic, Isbel Flores, Lizet, Estéfani y Héctor comentaron que la Central Obrera Boliviana (COB) nunca ha sido un aliado de las organizaciones NNAT ni nacional ni internacionalmente. Sospechan que, más allá de tener una visión del niño que no les corresponde, la COB considera que no es en su interés de promover el trabajo de los NNAT, que ocupan un espacio significativo en el mercado laboral.

2 Entrevista personal con Lizet en La Paz, 11/09/2018.
} 
NNAT desde los años 1980. Tales estructuras proporcionaron comida, espacio y conocimiento a nińos, niñas y adolescentes trabajadores en área urbanas.

En estos espacios de intercambios diversos, siguiendo clases y creando vínculos interpersonales, se creó una conciencia de pertenencia a un mismo grupo social bajo representaciones comunes, a las cuales se agregó la importancia dada por la fundación a la autonomía y la participación de los niños, niñas y adolescentes, algunos NNAT crearon en los años 1990 una organización de niños, niñas y adolescentes trabajadores, Taypinats. En esta organización, se cristalizó la identidad NNAT, y la misma lógica se ha observado hasta hoy. Un NNAT tiene menos de dieciocho ańos, trabaja, estudia, apoya a su familia y a sus compañeros y lucha "por un trabajo digno" - es decir, simultáneamente por sus derechos de niños y sus derechos de trabajadores. Esto crea una dinámica nueva, donde los derechos de los niños, y de los trabajadores no se contradicen y se deben adaptar a la situación específica de los NNAT.

Además, por un lado, la lucha de los NNAT se inscribió en luchas más amplias de movimientos sociales de fines del siglo XIX y de inicios de los ańos 2000 que luchaban contra un sistema productor de injusticia y desigualdades. Cabe notar que la historia política de la sociedad boliviana se caracteriza por un papel primordial de los movimientos sociales y una organización de la sociedad civil fuerte. Esta lógica se encuentra hasta hoy. Por ejemplo, la Encuesta Mundial de Valores de 2017 mostró que «la participación en organizaciones sociales es significativa en Bolivia, la más alta de América Latina, y una de la más altas del mundo» (Centro de Investigaciones Sociales (CIS) y Ciudadanía/Comunidad de Estudios Sociales y Acción Pública, 2017, p. 33).

La llegada de Evo Morales al poder creó condiciones favorables para que las organizaciones NNAT de varios departamentos del país pudieran expresar su identidad y sus demandas a través de una movilización nacional que culminó en 2013 con las manifestaciones de la Unatsbo, en La Plaza Murillo de La Paz. Queda no obstante aclarar los procesos exactos de la creación de la organización nacional en 2003. En estos espacios de luchas, se fortaleció la identidad NNAT como sujeto político y actor de su sociedad, o protagonista.

El contexto boliviano se caracteriza por representaciones de la infancia y del trabajo diferentes a los países occidentales. Los NNAT luchan vigorosamente contra la explotación económica, y tienen una visión del trabajo positiva cuando este no genera daños físicos y les permite tener tiempo para estudiar. El trabajo tiene un valor de ayuda, de contribución a la comunidad y se considera a menudo importante en el desarrollo personal del individuo. Estos dos puntos contradicen las representaciones difundida por organizaciones internacionales, bajo las cuales infancia y educación de un lado, y trabajo del otro, se excluyen mutuamente. La oposición de 
organizaciones como la OIT demuestra una presencia muy fuerte de las representaciones occidentales, basadas en una cultura e historia mayormente europea, en el derecho internacional. Estas normas aspiran a aplicarse universalmente, olvidando que los derechos humanos, aunque necesarios para fijar estándares, garantizar dignidad y libertades fundamentales, se entienden y se expresan de manera diferente a través de las sociedades y de las culturas.

En un ensayo escrito en 2010 con NNAT bolivianos llamado Mi fortaleza es mi trabajo. De las demandas a la propuesta. niños, niñas y adolescentes trabajadores y la regulación del trabajo infantil y adolescente en Bolivia, se puede leer:

Tal vez ya no sean importantes las divisiones, ni las categorizaciones de trabajo infantil, sino la apertura a escuchar sus sensaciones, opiniones y demandas que surgen ahora, y son tan antiguas como su lucha por erradicarlos; de lo contrario, nuevamente estaríamos cayendo en el riesgo de la categorización y esto resulta peligroso, pues a veces se confunden las categorías, si los quehaceres se parecen, a veces, los contextos pueden ser diferentes (Vargas Vallejo \& Unatsbo, 2010, p. 30).

Como lo muestra la situación de los NNAT, quién crea las categorías, según qué representaciones y en qué contexto, tiene una importancia primordial no solamente en modelar los discursos, sino también en el diseño de leyes y políticas públicas. De hecho, «el derecho es simultáneamente una representación social y un producto cultural» (Cussiánovich, 2010, p. 50). El Alto Comisionado para los Derechos Humanos de la ONU escribió: «los derechos humanos son reconocidos universalmente a pesar de diferencias culturales, pero su implementación práctica requiere una sensibilidad a cada cultura» (OHCHR, 2006, p. 5). Agregó que «desde una perspectiva de derechos humanos, la participación va mucho más allá de la simple constatación o de una consultación técnica en el diseño de proyecto. La participación debería ser vista como necesaria para promover la conciencia crítica y la toma de decisión como bases para una ciudadanía activa» (Vargas Vallejo \& Unatsbo, 2010, p. 26).

El movimiento social de los NNAT bolivianos, y de manera más general de los NNAT latinoamericanos, muestra que tenemos que considerar a los niños, niñas y adolescentes como agentes activos, como protagonistas, que tienen un papel mayor en definir su propia vida. Por lo tanto, no se pueden ver exclusivamente como futuros adultos e individuos en desarrollo, sino también como actores sociales en el presente y, potencialmente, actores políticos. Por lo tanto, los NNAT, y de manera general todos los niños, niñas y adolescentes, no se pueden ignorar más. Tienen el derecho a una participación real en los debates y la toma de decisión que afectan sus vidas y así, se deben tomar en cuenta en las tomas de decisiones y la elaboración tanto de leyes como de políticas públicas que tienen un impacto en su vida, que sea a escala local, nacional o internacional. 


\section{REFERENCIAS}

Agrikoliansky, E. \& Fillieule, O. (2010). Penser les mouvements sociaux. París: La Découverte. Alliance 8.7 (2017). 2017 Global Estimates of Modern Slavery and Child Labour: Regional brief for the Americas. Recuperado de http://www.ilo.org/global/topics/child-labour/WCMS_597871/lang--en/index.htm

Alonso, A. (2012). Repertório, Segundo Charles Tilly: História de um Conceito. Sociologia e Antropologia, 2(3), 21-41.

Benford, R. D. \& Snow, D. A. (2000). Framing Processes and Social Movements: An Overview and Assessment. Annual Review of Sociology, 26, 611-639. https://doi. org/10.1146/annurev.soc.26.1.611

Bonnet, M.\& Schlemmer, B. (2009). Aperçus sur le travail des enfants. Mondes en développement, 146(2), 11-25. https://doi.org/10.3917/med.146.0011

Bourdillon, M. (2006). Children and Work: A Review of Current Literature and Debates. Dévelopment and Changes, 37(6), 1201-1226. https://doi. org/10.1111/j.1467-7660.2006.00519.x

Cavagnoud, R. (2014). 13. Travail des enfants et économie informelle: le cas du Pérou, La Découverte, 14(1), 216-228. https://doi.org/10.3917/rce.014.0216

Cavagnoud, R., de Suremain, C-E. \& La Riva González, P. (2013). Infancia y niños en las sociedades andinas contemporáneas. Bulletin de l'Institut Français d'Études Andines, 43(2), 323-585.

Centro de Investigaciones Sociales (CIS) y Ciudadanía, Comunidad de Estudios Sociales y Acción Pública (2017). Encuesta Mundial de Valores en Bolivia 2017. La Paz: Vicepresidencia del Estado Plurinacional de Bolivia, Centro de Investigaciones Sociales (CSI).

Coalición Boliviana por los Derechos de los Niños, Niñas y Adolescentes (2018). Diagnóstico sobre la situación actual de las niñas, niños y adolescentes en Bolivia. La Paz, Bolivia.

Comunidad de Derechos Humanos (2015). Recomendaciones al Estado Plurinacional de Bolivia sobre los Derechos de Niñas, Niños y Adolescentes Realizadas en el Examen Periódico Universal (EPU) de Bolivia. 2014, Bolivia.

Cuche, D. (2016) La notion de culture dans les sciences sociales (5e éd.). París: La Découverte. https://doi.org/10.3917/dec.cuche.2016.01

Cussiánovich, A. (2010). Ensayo sobre infancia. Sujeto de derechos y protagonista. Lima: IFEJANT.

Dómic, J. (1999). Niños trabajadores: la emergencia de nuevos actores sociales. La Paz: PIEB.

Dumoulin Kervran, D. (2006) Les ONG latino-américaines après l'âge d'or: internationalisation et dispersion. En P. Zagefka (dir.), Amérique Latine 2006 (pp. 31-50). París: La Documentation Française, Paris.

Fillieule, O., Mathieu, L. \& Péchu, C. (dir.) (2009). Dictionnaire de mouvements sociaux. París: Presses de Sciences Po. 
García Linera, Á. (coord.), Chávez León, M. \& Costas Monje, P. (2010). Sociología de los Movimientos Sociales en Bolivia. Estructuras de movilización, repertorios culturales y acción política, cuarta edición. La Paz: Plural.

Goirand, C. (2010). Penser les mouvements sociaux en Amérique Latine. Les approches des mobilisations depuis les années 1970. Revue française de science politique, 60(3), 445466. https://doi.org/10.3917/rfsp.603.0445

González Ortega, N. (2017). Bolivia en el Siglo XXI. Trayectorias históricas y proyecciones politicas, económicas y socioculturales. La Paz: Plural Editores / Universidades de Oslo e Iberoamericana Vervuert. https://doi.org/10.31819/9783964566584

Holzscheiter, A. (2016). Representation as power and performative practice: Global civil society advocacy for working children. Review of International Studies, 42, 205-226. https://doi.org/10.1017/S0260210515000145

Invernizzi, A. (2003). Des enfants libérés de l'exploitation ou des enfants travailleurs doublement discriminés? Positions et oppositions sur le travail des enfants. Déviance et Société, 27(4), 459-481. https://doi.org/10.3917/ds.274.0459

Liebel, M. (2004). A Will of Their Own. Cross-Cultural Perspectives on Working Children. Londres/Nueva York: Zed Books.

McAdam, D., Tarrow \& S., Tilly, C. (2008). Methods for Measuring Mechanisms of Contention. Qualitative Sociology, 31(4), 307-331. https://doi.org/10.1007/ s11133-008-9100-6

Mead, M. (1926). Coming of Age in Samoa. Victoria: Penguin Books.

Morais de Ramírez, M. G. (2016). El Nuevo Código Niña, Niño y Adolescente. ¿Reflejo de la Convención sobre Derechos del Niño? En M. G. Morais de Ramírez (coord.), Aportes al Código Niña, Niño y Adolescente Ley 548. Cochabamba: Unicef - Universidad Católica Boliviana San Pablo.

Morsolin, C. (2017). Trabajo Infantil y politicas públicas emancipadoras en Bolivia. Bogotá: SELVAS.

Morsolin, C. (2009). "Protagonisme» de mouvements d'enfants travailleurs en Amérique Latine. Alternatives Sud, 16, 161-175.

OHCHR (2006). Frequently Asked Questions on a Human Rights-Based Approach to Development Cooperation. Nueva York / Ginebra: United Nations.

Okyere, S. (2013). Are Working Children Rights and Child Labour Abolition Complementary or Opposing Realms? International Social Work, 56(1), 80-91.

Pochetti, I. (2018). Introduction. Enfances, Droits et Politique. Problèmes d'Amérique Latine, 108(1), 5-18. https://doi.org/10.3917/pal.108.0005

Rateau, P., Molinier, P., Guimelli, C. \& Abric, J-C. (2012). Social Representation Theory. Handbook of Theories of Social Psychology, 50, 477-497, https://doi. org/10.4135/9781446249222.n50

Salazar, M. C. (1998). Child Work and Education in Latin America. The International Journal of Children's Rights, 6, 155-177. https://doi.org/10.1163/15718189820494012

Silva, E. (2009). Challenging Neoliberalism in Latin America. Nueva York: Cambridge University Press. https://doi.org/10.1017/CBO9780511803222 
Tilly, C. \& Tarrow, S. (2008). Politique(s) du Conflit. De la grève à la révolution. París: Presses de Sciences Po.

Tilly, C. \& J. Wood, L. (2009). Social Movements as Politics. En C. Tilly y J. Wood, Social Movements 1768-2008 (pp. 1-15). Boulder, CO: Paradigm Publishers.

Truchon, K. \& Saillant, F. (2013). Droits et cultures en mouvement. Quebec: Presses de l'Université Laval.

Vargas Vallejo, P. (ed.) y Unatsbo (2010). Mi fortaleza es mi trabajo. De las demandas a la propuesta. niños, niñas y adolescentes trabajadores y la regulación del trabajo infantil y adolescente en Bolivia. Cochabamba, Bolivia. 